\section{Breeding and Evaluation of Fine Fescues for Increased Tolerance to Mesotrione Herbicide}

\author{
Trent M. Tate ${ }^{1}$, Stacy A. Bonos ${ }^{1}$, and William A. Meyer ${ }^{1}$
}

Additional IndeX wORDs. annual bluegrass control, Festuca, Festuca rubra ssp. commutate, Festuca brevipila, F. rubra ssp. rubra, herbicide injury, recurrent selection

SUMMARY. Fine fescues (Festuca sp.) are a group of species that require fewer inputs, such as fertilizer, than other cool-season species managed for turf. They are adapted to infertile, acidic soils; shade; and drought. One area that poses additional challenges is the lack of weed control options for fine fescues during establishment from seed. Mesotrione is a herbicide that provides preemergence control of many broadleaf and grassy weeds, such as annual bluegrass (Poa annua), but is currently not labeled for use in fine fescues at seeding. The objectives of this research were 1) to use a recurrent selection technique to develop mesotrione-tolerant chewings fescue (Festuca rubra ssp. commutata), hard fescue (Festuca brevipila), and strong creeping red fescue (F. rubra spp. rubra); and 2) to conduct field trials to compare the new selections to commercially available cultivars and experimental lines not selected for tolerance to mesotrione. Progress was made after each of the three generations of recurrent selection. The top statistical grouping of entries for injury following application of mesotrione at the 8-oz/acre rate included all the thirdgeneration (G3) hard fescues, all the G3 chewings fescues, and the G3 strong creeping red fescue STB1 Composite. After three generations, selections of hard, chewings, and strong creeping red fescues had equivalent or better tolerance to mesotrione than tall fescue (Festuca arundinacea) and kentucky bluegrass (Poa pratensis) cultivars, which are on the label for safe use at seeding. These new selections would provide turf managers an option to control weeds using mesotrione during seedling establishment of fine fescues.

$\mathrm{F}$ ine fescues (Festuca sp.) are a group of cool-season turfgrass species that have a needle-like fine leaf texture and are well adapted to cool, humid regions of the world. They are also adapted to infertile, acidic soils; shade; and drought (Beard, 1973; Hanson et al., 1969; Turgeon, 1996). This group of species does well under lower fertility compared with other cool-season grasses (Ruemmele et al., 2003). The fine fescues have been found in a wide range of habitats, from beaches, dunes, coastal rock, cliffs, salt marshes, meadows, and grasslands (Pavlick, 1985). These traits

Received for publication 4 Dec. 2020. Accepted for publication $16 \mathrm{Feb} .2021$

Published online 14 April 2021

${ }^{1}$ Department of Plant Biology, Rutgers, The State University of New Jersey, 59 Dudley Road, Foran Hall, New Brunswick, NJ 08901

We thank Ruying Wang, Ron Bara, Dirk Smith, Phillip Vines, and Ryan Daddio for their tremendous help with this research.

T.M.T. is the corresponding author. E-mail: trentmtate@gmail.com.

This is an open access article distributed under the CC BY-NC-ND license (https://creativecommons.org/ licenses/by-nc-nd $/ 4.0 /$ ).

https://doi.org/10.21273/HORTTECH04772-20 make them good choices for lowmaintenance areas (Beard, 1973; Meyer and Funk, 1989; Turgeon, 1996). Once established, these species need few inputs to maintain a good turf stand.

Currently, there are very few options for preemergence weed control during seeding and establishment of fine fescues. Preemergence weed control before and during establishment is an important component to establish successfully a healthy stand of cool-season turfgrass (Beard, 1973; Musser and Perkins, 1969). Weeds compete for light, water, and nutrients, and usually have a much faster establishment and growth rate than fine fescues. The recommended timing for turfgrass establishment in New Jersey is 15 Aug. to 5

\begin{tabular}{llll}
\hline $\begin{array}{l}\text { Units } \\
\begin{array}{l}\text { To convert U.S. to SI, } \\
\text { multiply by }\end{array}\end{array}$ & U.S. unit & SI unit & $\begin{array}{l}\text { To convert SI to U.S., } \\
\text { multiply by }\end{array}$ \\
\hline 0.3048 & $\mathrm{ft}$ & $\mathrm{m}$ & 3.2808 \\
9.3540 & $\mathrm{gal} / \mathrm{acre}$ & $\mathrm{L} \cdot \mathrm{ha}^{-1}$ & 0.1069 \\
2.54 & inch $(\mathrm{es})$ & $\mathrm{cm}$ & 0.3937 \\
48.8243 & $\mathrm{lb} / \mathrm{l} 000 \mathrm{ft}^{2}$ & $\mathrm{~kg} \cdot \mathrm{ha}^{-1}$ & 0.0205 \\
1.1209 & $\mathrm{lb} / \mathrm{acre}$ & $\mathrm{kg} \cdot \mathrm{ha}^{-1}$ & 0.8922 \\
70.0532 & $\mathrm{oz} / \mathrm{acre}$ & $\mathrm{g} \cdot \mathrm{ha}^{-1}$ & 0.0143
\end{tabular}

Oct. (Grande, 2004). This timing coincides with the peak emergence of annual bluegrass [Poa annua (Kaminski and Dernoeden, 2007)], which is what makes it the most problematic weed to control when establishing new cool-season turfgrasses in the northeastern United States. Having safe, selective preemergence control of annual bluegrass in fine fescues would increase the ability to establish these lowmaintenance grasses successfully. Mesotrione is a 4-hydroxyphenylpyruvate dioxygenase-inhibiting herbicide that controls many monocot and dicot weeds selectively at seeding in many cool-season turfgrasses (Askew and Beam, 2002; Hart et al., 2007; Willis et al., 2006). Currently, mesotrione is the active ingredient in Tenacity (Syngenta Crop Protection, Greensboro, NC) which is labeled for use in many cool-season turfgrasses at rates of $2-4 \mathrm{oz} /$ acre mesotrione. It is not currently labeled for use in fine fescues at seeding or for use in seed blends that contain more than $20 \%$ fine fescue (Syngenta Crop Protection, 2008). Mesotrione effectively provides preemergence control of both broadleaf and grassy weeds. Mesotrione can have several negative effects on fine fescues. Phototoxicity (bleaching of leaf tissue) is commonly associated with the use of mesotrione on fine fescue (Williams et al., 2009). In the study conducted by Williams et al. in 2009, they concluded that no rate or timing used in the study was safe to use on chewings fescue at seeding. These effects can impact significantly the ability of fine fescue seedlings to establish and survive.

There are previous examples of using recurrent selection in breeding fine fescues for increased tolerance to herbicides. Johnston and Faulkner (1986) developed the aminotriazoletolerant 'Countess' chewings fescue to control annual bluegrass. Herbicide tolerance development in hard 
fescue has been demonstrated previously with the nonselective herbicide glyphosate to develop 'Aurora Gold', which is an advanced-generation synthetic cultivar derived from Aurora hard fescue. Five cycles of phenotypic recurrent selection over a 10-year period after direct applications of glyphosate at 0.8 to 1.6 $\mathrm{kg} \cdot \mathrm{ha}^{-1}$ was used to develop this cultivar (Hart et al., 2005). Further study of 'Aurora Gold' by McCullough et al. (2015) determined the mechanism of resistance to glyphosate was due the result of lower target-site inhibition. The successful establishment of fine fescues is greatly influenced by controlling weeds.

Having mesotrione-tolerant fine fescues would give turf managers an option to control problematic weeds during establishment. The objectives of this research were 1) to use a recurrent selection technique (Vogel and Pedersen, 1993) to develop mesotrione-tolerant chewings, hard, and strong creeping red fescues; and 2) to conduct field trials to compare the new selections to commercially available cultivars and experimental lines not selected for tolerance to mesotrione.

\section{Materials and methods}

First generation. All research for this project was conducted at the at the Rutgers Plant Biology Research and Extension Station in Adelphia, NJ. Soil type was a Holmdel sandy loam (fine-loamy, mixed, active Aquic Hapludults) with a $\mathrm{pH}$ of 6.7 and $2.2 \%$ organic matter. Plant selections were made from a spaced-plant nursery that had been sprayed with three applications of mesotrione at a rate of $4 \mathrm{oz} /$ acre $+0.25 \% \mathrm{v} / \mathrm{v}$ nonionic surfactant (Activator 90; Loveland Products, Greeley, CO) at 4-week intervals during late Summer/early Fall 2011. Applications were made with a tractor-mounted agricultural sprayer set to deliver $30 \mathrm{gal} / \mathrm{acre}$. Irrigation was applied only if there was no rainfall event within $3 \mathrm{~d}$ of application. Plants that had no bleaching injury response to those applications were noted and, the following spring, were grouped by growth habit, color, leaf texture, and flowering time into crossing blocks before anthesis. In total, 189 plants were moved in May 2013 into seven different crossing blocks: two hard fescue, three chewings, and two strong creeping red fescue. Individual plants were harvested when seeds were mature. The harvested material was then dried and threshed, and a composite for each block was made using equal amounts of seed from each plant in the block.

Two field trials were planted to determine the herbicide effects of the first generation (Gl) of selections on 9 Sept. 2013 and 18 Sept. 2014. The 2013 turf trial included a replicated section that included a composite of each crossing block, commercially available cultivars, and experimental lines that had not undergone mesotrione selection. A nonreplicated section of single plot progenies from each maternal parent of the seven crossing blocks described previously was also included. This nonreplicated section of the trial is used only for breeding and selection work and is not part of any statistical analysis because there is no replication. The unreplicated progeny plots were included in the trial to observe the performance of the progeny from each maternal parent and to make selections to tiller plant material for further selection work and for breeding subsequent generations. Plots measured $3 \times 5 \mathrm{ft}$, with a 6 -inch unseeded border, and were sown at a rate of $3.67 \mathrm{lb} / 1000 \mathrm{ft}^{2}$. A randomized complete block design was used with three replications. The 2014 trial was a repeat of the 2013 , but only included the replicated entries. Trials were maintained at a 2.5 -inch mowing height with rotary mower at a frequency to avoid excessive accumulation of clippings. Each trial received $1 \mathrm{lb} / 1000 \mathrm{ft}^{2}$ of nitrogen $(\mathrm{N})$ at seeding as $10 \mathrm{~N}-4.4 \mathrm{P}-8.3 \mathrm{~K}$ and an additional $2 \mathrm{lb} / 1000 \mathrm{ft}^{2} \mathrm{~N}$ as $19 \mathrm{~N}-$ $0 \mathrm{P}-7.5 \mathrm{~K}$ over the life of the trial $(2$ years) in two $0.5-\mathrm{lb} / 1000 \mathrm{ft}^{2}$ applications per year of the trial. Both trials were irrigated to prevent severe drought stress during the summer months.

Applications of mesotrione were made after sowing at a rate of $3 \mathrm{oz} /$ acre $+0.25 \% \mathrm{v} / \mathrm{v}$ nonionic surfactant followed by an application of $2 \mathrm{oz} /$ acre $+0.25 \% \mathrm{v} / \mathrm{v}$ nonionic surfactant $28 \mathrm{~d}$ after seedling emergence to the entire field trial area. Visual ratings were taken for establishment, injury through the first 12 weeks after planting, and quality for a 2-year period after the trial was planted. Each visual rating used a 1- to 9-point scale, where 9 is the best establishment, least amount of injury, and highest turf quality, respectively. For establishment, injury, and turf quality, the minimum acceptable rating is 5 . Data were subjected to analysis of variance with the General Linear Model procedure in SAS (version 9.3; SAS Institute, Cary, NC). Means were separated with Fisher's protected least significant difference (LSD) test at $\alpha=0.05$.

SECOND GENERATION. Individual plants from the single plot progenies from the 2013 trial were selected from plots with the least mesotrione injury and best turf-quality ratings. Tillers were taken and individual plants were planted in a spaced-plant nursery and allowed to establish. In total, there were 6840 plants planted in the nursery: 3456 hard fescues, 2616 chewings fescues, and 768 strong creeping red fescues. Four applications of mesotrione at $4 \mathrm{oz} /$ acre at 4-week intervals were made, followed by an application at $5 \mathrm{oz} /$ acre 2 weeks after the last 4 -oz/acre application made during late Summer/early Fall 2014, as described previously for the Gl field application. Bleaching injury symptoms began to appear 3 to $5 \mathrm{~d}$ after the last application. Plants exhibiting no bleaching injury were documented. The following spring, plants that exhibited no injury and had good turf quality traits with no disease were selected and grouped by growth habit, color, leaf texture, and flowering time, and were moved into crossing blocks before anthesis. In total, 290 plants were moved into eight individual crossing blocks: two chewings, four hard, and two strong creeping red fescues. Individual plants were harvested, dried, and threshed, and a composite for each block was made using seed from each plant in the block.

The second-generation (G2) turf trial (2015) included both a replicated section and single plot progeny as described for the 2013 trial. The turf trial was seeded 26 Aug. 2015. This trial consisted of $4-\times 6$ - $\mathrm{ft}$ plots, with a 6 -inch unseeded border, and were sown at a rate of $3.67 \mathrm{lb} / 1000 \mathrm{ft}^{2}$. Larger plots were used so herbicide strip treatments could be applied. The strips included a 2 -ft strip of a 3-oz/ 
acre rate of mesotrione and a $2-\mathrm{ft}$ strip of nontreated control. An example of two plots with each strip is shown in Fig. 1. Each treatment was applied at sowing and was repeated 28 $\mathrm{d}$ after seedling emergence with a carbon dioxide $\left(\mathrm{CO}_{2}\right)$-pressurized sprayer calibrated to deliver $30 \mathrm{gal} / \mathrm{acre}$. Plots were arranged in a randomized complete block design with three replications. Ratings were taken as described previously for injury and turf quality. An additional rating capturing the negative impact to establishment from the mesotrione treatment was also included. This was a comparison of the mesotrione strip to the nontreated portion of the turf plot. Ratings were made on a 1 to 9 -scale, where 9 is no negative effect on establishment from mesotrione and 1 is complete inhibition of establishment. Data were subjected to analysis of variance with the General Linear Model procedure in SAS (version 9.3). Means were separated with Fisher's protected LSD test at $\alpha=$ 0.05 . Trial maintenance, including mowing, fertility, and irrigation, are the same as described previously for the Gl trials.

Third generation. Selections from the single plot progeny section of the 2015 trial were made based on mesotrione-induced injury, effect of mesotrione on establishment, and overall turf quality for the following year. In total, 7692 individual selections were planted in Fall 2016: 3900 hard fescues, 2112 chewings fescues, and 1680 strong creeping red fescues. Two applications of mesotrione at a rate of $6 \mathrm{oz} / \mathrm{acre}+0.25 \% \mathrm{v} / \mathrm{v}$ nonionic surfactant at a 2 -week interval followed by an application of mesotrione at a rate of $8 \mathrm{oz} /$ acre +

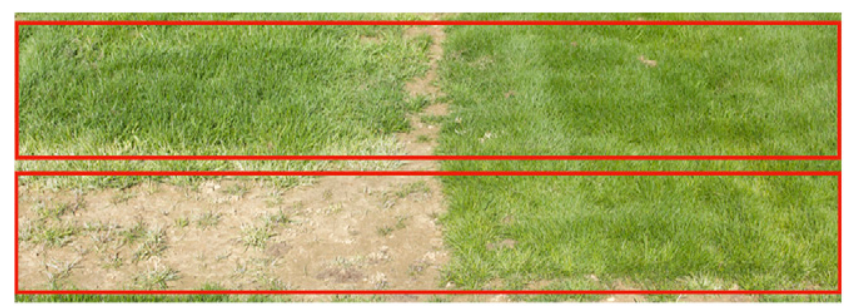

Fig. 1. Example of mesotrione-treated strip (bottom) and a nonmesotrionetreated strip (top) in a field trial including first-, second-, and third-generation mesotrione-tolerant selections, commercially available cultivars, and experimental selections of fine fescues. Note the bleaching injury and decreasing establishment in a nonmesotrione selected fine fescue turf plot (left) and a third-generation mesotrione-tolerant selection turf plot (right) exhibiting no injury or reduction in establishment.
$0.25 \% \mathrm{v} / \mathrm{v}$ nonionic surfactant 1 week after the previous spray. Sprays were conducted as described for Gl field applications. Increased rates of mesotrione and a shorter reapplication interval were necessary because no injury was observed after the two 6$\mathrm{oz} /$ acre rate applications at a 2 -week interval. Injury was recorded and plants were selected the following spring using the parameters described previously. In total, 314 plants were selected to use in 14 individual crossing blocks: five chewings fescues, five hard fescues, and four strong creep-

The G3 selected plants were planted in a field trial seeded on 5 Sept. 2018. Plots were $3 \times 5 \mathrm{ft}$, with a 6 -inch unseeded border, and were sown at a rate of $3.67 \mathrm{lb} / 1000 \mathrm{ft}^{2}$. A randomized complete block design was used with three replications for the replicated entries, followed by an unreplicated section of single plot progeny of each maternal line of the crossing blocks. Replicated plots had three 1.5-ft-wide strip treatments: $1.5 \mathrm{ft}$ of $4 \mathrm{oz} /$ acre mesotrione + $0.25 \% \mathrm{v} / \mathrm{v}$ nonionic surfactant, 1.5 $\mathrm{ft}$ of untreated control, and $1.5 \mathrm{ft}$ of 8 $\mathrm{oz} /$ acre mesotrione $+0.25 \% \mathrm{v} / \mathrm{v}$ nonionic surfactant. Each treatment was applied at sowing and at $28 \mathrm{~d}$ after seedling emergence with a $\mathrm{CO}_{2}$-pressurized sprayer calibrated to deliver $30 \mathrm{gal} / \mathrm{acre}$. Plots were rated for injury and establishment inhibition as a comparison with the untreated control section of the same plot. The rating was made based on a 1 - to 9 scale, where 9 is no injury or reduction in establishment and $\mathrm{l}$ is no establishment or all bleached tissue. There were also tall fescue (Festuca arundinacea) and kentucky bluegrass ing red fescues.
(Poa pratensis) cultivars included in our study to compare the mesotrione selected fine fescue material with species that are on the Tenacity label and considered safe to use at seeding. Ratings were taken separately for the 4 - and $8-\mathrm{oz} /$ acre rate strips. Data were subjected to analysis of variance with the General Linear Model procedure in SAS (version 9.3). Means were separated with Fisher's protected LSD test at $\alpha=0.05$. Trial maintenance, including mowing, fertility, and irrigation, are the same as described for the Gl trials.

\section{Results and discussion}

Gl Field trial. The mesotrione injury ratings for the Gl trials ranged from 5.8 to $2.0($ LSD $=1.0)$. Hard fescues overall exhibited the most injury to mesotrione compared with chewings and strong creeping red fescues. Both Gl mesotrione-tolerant hard fescue selections (MEHI Composite and $\mathrm{MEH} 2$ Composite) were in the top statistical grouping for having the lowest injury, with ratings of 4.5 and 4.3 , respectively (Table 1 ). Chewings fescue, as a species, exhibited the least injury to mesotrione compared with strong creeping red fescue and hard fescue. The Gl mesotrione-tolerant composites of chewings fescue (MEWl Composite, MEW2 Composite, and MEW3 Composite) exhibited light to moderate injury, with ratings of $4.8,5.8$, and 5.0, respectively. However, 'Radar' chewings fescue also rated a 4.8 for mesotrione injury. The strong creeping red fescues were between hard and chewings fescue for mesotrione injury. The Gl mesotrionetolerant selection composites of strong creeping red fescue (MESI Composite and MES2 Composite) exhibited moderate injury, with ratings of 3.7 and 3.3, respectively. Each of the Gl mesotrione-tolerant selection composites had lower mesotrione injury (higher ratings) and were near the top of the ratings for each of their species group compared with experimental selections and commercial cultivars, except for strong creeping red fescue.

Establishment for the trial had a range of 5.8 to $1.4(\mathrm{LSD}=1.0)$. The two Gl mesotrione-tolerant selection hard fescues (MEHl Composite and $\mathrm{MEH} 2$ Composite) were somewhat slower to establish; therefore, the 
Table 1. Establishment and mesotrione injury ratings of first-generation mesotrione-tolerant selections, experimental selections, and commercially available cultivars of fine fescue in two field trials planted in Sept. 2013 and Sept. 2014 at the Rutgers Plant Biology Research and Extension Station in Adelphia, NJ. Mesotrione was applied at sowing (3 oz/acre + $0.25 \% \mathrm{v} / \mathrm{v}$ nonionic surfactant $)$ and $28 \mathrm{~d}$ after seedling emergence $(2 \mathrm{oz} / \mathrm{acre}+0.25 \% \mathrm{v} / \mathrm{v}$ nonionic surfactant $)$. Data were subjected to analysis of variance with the General Linear Model procedure in SAS (version 9.3; SAS Institute, Cary, NC). Means were separated with Fisher's protected least significant difference (LSD) test at $\alpha=0.05 .\left(1 \mathrm{oz} / \mathrm{acre}^{2}=70.0532 \mathrm{~g} \cdot \mathrm{ha}^{-1}\right)$.

\begin{tabular}{|c|c|c|c|c|}
\hline Injury rank & Entry $^{z}$ & Species & Establishment (1-9 scale $)^{y}$ & Injury $(1-9 \text { scale })^{x}$ \\
\hline 1 & MEW2 Composite (Gl) & Chewings & 5.3 & 5.8 \\
\hline 2 & Seabreeze GT & Slender creeping & 4.4 & 5.2 \\
\hline 3 & MEW3 Composite (Gl) & Chewings & 4.9 & 5.0 \\
\hline 4 & $4-12 \mathrm{FF}-1$ & Strong creeping & 5.8 & 5.0 \\
\hline 5 & MEWl Composite (Gl) & Chewings & 5.2 & 4.8 \\
\hline 6 & Radar & Chewings & 4.9 & 4.8 \\
\hline 7 & 7W4 Composite & Chewings & 4.2 & 4.7 \\
\hline 8 & $4-12 \mathrm{FF}-2$ & Strong creeping & 4.4 & 4.7 \\
\hline 9 & MEHl Composite (Gl) & Hard & 2.8 & 4.5 \\
\hline 10 & Daisy & Sheep & 2.5 & 4.5 \\
\hline 11 & 2 -10FRR-12 & Strong creeping & 4.2 & 4.5 \\
\hline 12 & $2-10$ FRR-13 & Strong creeping & 4.2 & 4.5 \\
\hline 13 & $5-12 \mathrm{FF}-5$ & Strong creeping & 4.5 & 4.5 \\
\hline 15 & PPG-FRC 113 & Chewings & 4.0 & 4.3 \\
\hline 16 & PPG-FRC 115 & Chewings & 4.6 & 4.3 \\
\hline 17 & PPG-FRC 107 & Chewings & 4.8 & 4.3 \\
\hline 14 & MEH2 Composite (G1) & Hard & 3.0 & 4.3 \\
\hline 18 & 4-12FF-BULK & Strong creeping & 5.2 & 4.3 \\
\hline 19 & $4-12 \mathrm{FF}-5$ & Strong creeping & 5.2 & 4.3 \\
\hline 20 & Fairmont & Chewings & 4.8 & 4.2 \\
\hline 23 & PPG-FRC 114 & Chewings & 5.0 & 4.0 \\
\hline 25 & Shadow II & Chewings & 3.9 & 4.0 \\
\hline 26 & SR5130 & Chewings & 4.4 & 4.0 \\
\hline 27 & $08-5 \mathrm{FCE}+$ & Chewings & 3.5 & 4.0 \\
\hline 28 & Ambrose & Chewings & 2.8 & 4.0 \\
\hline 29 & 7W2 Composite & Chewings & 3.8 & 4.0 \\
\hline 21 & TEl Composite & Hard & 2.3 & 4.0 \\
\hline 22 & BM2 Composite & Hard & 1.9 & 4.0 \\
\hline 24 & $2-10$ FRR- 8 & Strong creeping & 4.6 & 4.0 \\
\hline 30 & PPG-FRR 111 & Strong creeping & 4.5 & 4.0 \\
\hline 31 & $4-12 \mathrm{FF}-3$ & Strong creeping & 4.9 & 4.0 \\
\hline 36 & 7W3 Composite & Chewings & 3.6 & 3.8 \\
\hline 32 & H575 Composite & Hard & 2.3 & 3.8 \\
\hline 33 & PSG TH3 & Hard & 3.4 & 3.8 \\
\hline 34 & 7H3 Composite & Hard & 2.4 & 3.8 \\
\hline 35 & TE2 Composite & Hard & 1.7 & 3.8 \\
\hline 37 & FT6 Composite & Strong creeping & 2.8 & 3.8 \\
\hline 38 & $5-12 \mathrm{FF}-8$ & Strong creeping & 4.4 & 3.8 \\
\hline 39 & FF2 & Strong creeping & 4.3 & 3.8 \\
\hline 40 & 4GRP & Strong creeping & 3.5 & 3.8 \\
\hline 47 & Compass & Chewings & 4.9 & 3.7 \\
\hline 41 & H573 Composite & Hard & 2.3 & 3.7 \\
\hline 42 & Reliant IV & Hard & 2.3 & 3.7 \\
\hline 43 & 7Hl Composite & Hard & 2.8 & 3.7 \\
\hline 44 & PPG-FL 107 & Hard & 2.8 & 3.7 \\
\hline 49 & Seafire & Slender creeping & 3.0 & 3.7 \\
\hline 45 & MESl Composite (Gl) & Strong creeping & 3.2 & 3.7 \\
\hline 46 & Navigator II & Strong creeping & 4.4 & 3.7 \\
\hline 48 & CRF-11-4A & Strong creeping & 4.2 & 3.7 \\
\hline 50 & Shademaster III & Strong creeping & 3.2 & 3.7 \\
\hline 51 & $5-12 \mathrm{FF}-4$ & Strong creeping & 2.7 & 3.7 \\
\hline 54 & 4SHR-CH & Chewings & 4.5 & 3.5 \\
\hline 52 & Predator & Hard & 1.9 & 3.5 \\
\hline 53 & Cardinal & Strong creeping & 4.1 & 3.5 \\
\hline
\end{tabular}


Table 1. (Continued) Establishment and mesotrione injury ratings of first-generation mesotrione-tolerant selections, experimental selections, and commercially available cultivars of fine fescue in two field trials planted in Sept. 2013 and Sept. 2014 at the Rutgers Plant Biology Research and Extension Station in Adelphia, NJ. Mesotrione was applied at sowing (3 oz/ acre $+0.25 \% \mathrm{v} / \mathrm{v}$ nonionic surfactant $)$ and $28 \mathrm{~d}$ after seedling emergence $(2 \mathrm{oz} /$ acre $+0.25 \% \mathrm{v} / \mathrm{v}$ nonionic surfactant $)$. Data were subjected to analysis of variance with the General Linear Model procedure in SAS (version 9.3; SAS Institute, Cary, NC). Means were separated with Fisher's protected least significant difference (LSD) test at $\alpha=0.05$. $(1 \mathrm{oz} / \mathrm{acre}=70.0532$ g.ha $\left.{ }^{-1}\right)$.

\begin{tabular}{|c|c|c|c|c|}
\hline Injury rank & Entry ${ }^{z}$ & Species & Establishment (1-9 scale $)^{\mathrm{y}}$ & Injury $(1-9 \text { scale })^{x}$ \\
\hline 55 & FT2 Composite & Strong creeping & 3.0 & 3.5 \\
\hline 56 & Kent & Strong creeping & 4.3 & 3.5 \\
\hline 58 & 7Wl Composite & Chewings & 3.9 & 3.3 \\
\hline 57 & PPG-FL 106 & Hard & 2.4 & 3.3 \\
\hline 59 & 7C3 Composite & Strong creeping & 3.3 & 3.3 \\
\hline 60 & 2-10FRRBULK & Strong creeping & 4.1 & 3.3 \\
\hline 61 & MES2 Composite (G1) & Strong creeping & 3.6 & 3.3 \\
\hline 62 & SR5250 & Strong creeping & 4.0 & 3.3 \\
\hline 63 & OR 126 & Strong creeping & 3.8 & 3.3 \\
\hline 64 & PPG-FRR 103 & Strong creeping & 4.4 & 3.3 \\
\hline 65 & FTl Composite & Strong creeping & 3.1 & 3.3 \\
\hline 66 & PSG 5RM & Strong creeping & 2.9 & 3.3 \\
\hline 67 & BRSO & Strong creeping & 4.3 & 3.3 \\
\hline 68 & $5-12 \mathrm{FF}-6$ & Strong creeping & 3.8 & 3.3 \\
\hline 69 & BRSG & Strong creeping & 4.1 & 3.3 \\
\hline 70 & 5-12FF-BULK & Strong creeping & 3.6 & 3.3 \\
\hline 82 & PSG 50C3 & Chewings & 1.7 & 3.2 \\
\hline 85 & Windward & Chewings & 3.6 & 3.2 \\
\hline 71 & 7H4 Composite & Hard & 3.1 & 3.2 \\
\hline 72 & PPG-FL 108 & Hard & 3.7 & 3.2 \\
\hline 73 & H571 Composite & Hard & 2.2 & 3.2 \\
\hline 74 & 7H2 Composite & Hard & 1.8 & 3.2 \\
\hline 76 & 7H6 Composite & Hard & 3.1 & 3.2 \\
\hline 77 & Soilguard & Hard & 1.9 & 3.2 \\
\hline 75 & Marco Polo & Sheep & 3.8 & 3.2 \\
\hline 80 & Sealink & Slender creeping & 3.7 & 3.2 \\
\hline 78 & 2-10FRR-6 & Strong creeping & 4.3 & 3.2 \\
\hline 79 & S571 Composite & Strong creeping & 3.4 & 3.2 \\
\hline 81 & Jasper II & Strong creeping & 4.2 & 3.2 \\
\hline 83 & FT5 Composite & Strong creeping & 3.0 & 3.2 \\
\hline 84 & Gibraltar Gold & Strong creeping & 3.4 & 3.2 \\
\hline 86 & 4CRD-8 & Strong creeping & 3.5 & 3.2 \\
\hline 87 & Oracle & Strong creeping & 2.8 & 3.2 \\
\hline 91 & $4 \mathrm{CHY}$ & Chewings & 3.6 & 3.0 \\
\hline 89 & Oxford & Hard & 1.4 & 3.0 \\
\hline 88 & Blueray & Blue hard & 2.4 & 3.0 \\
\hline 90 & $2-10 F R R-4$ & Strong creeping & 4.2 & 3.0 \\
\hline 92 & FT3 Composite & Strong creeping & 2.5 & 3.0 \\
\hline 93 & 4CRD-P & Strong creeping & 3.5 & 3.0 \\
\hline 94 & Beacon & Hard & 3.0 & 2.8 \\
\hline 95 & Spartan II & Hard & 3.5 & 2.8 \\
\hline 96 & FRR 62 & Strong creeping & 2.8 & 2.8 \\
\hline 97 & Gibraltar & Strong creeping & 3.6 & 2.8 \\
\hline 98 & FT4 Composite & Strong creeping & 2.5 & 2.7 \\
\hline 99 & Miser & Strong creeping & 2.3 & 2.5 \\
\hline 100 & 4BND & Hard & 3.4 & 2.3 \\
\hline 101 & Bighorn GT & Hard & 1.9 & 2.3 \\
\hline \multirow[t]{2}{*}{102} & Azure & Hard & 1.8 & 2.0 \\
\hline & & $\operatorname{LSD}(\alpha=0.05)$ & 1.0 & 1.0 \\
\hline
\end{tabular}

${ }^{\mathrm{z}} \mathrm{Gl}$ in parentheses indicates first-generation mesotrione-tolerant selection.

${ }^{\mathrm{y}} 1=$ no establishment of seedlings (i.e., bare soil), $5=$ minimum acceptable rating, and $9=$ fully established turfgrass.

${ }^{\mathrm{x}} \mathrm{l}=$ completely bleached tissue, $5=$ minimum acceptable injury rating, and $9=$ no injury. 
Table 2. Overall, year 1 and year 2 turfgrass quality ratings of first-generation mesotrione-tolerant selections, experimental selections, and commercially available cultivars of fine fescue in two field trials planted in 2013 and 2014 at the Rutgers Plant Biology Research and Extension Station in Adelphia, NJ. Mesotrione was applied at sowing $(3 \mathrm{oz} / \mathrm{acre}+0.25 \% \mathrm{v} / \mathrm{v}$ nonionic surfactant) and $28 \mathrm{~d}$ after seedling emergence $(2 \mathrm{oz} /$ acre $+0.25 \% \mathrm{v} / \mathrm{v}$ nonionic surfactant $)$. Data were subjected to analysis of variance with the General Linear Model procedure in SAS (version 9.3; SAS Institute, Cary, NC). Means were separated with Fisher's protected least significant difference (LSD) test at $\alpha=0.05 .\left(1 \mathrm{oz} / \mathrm{acre}=70.0532 \mathrm{~g} \cdot \mathrm{ha}^{-1}\right)$.

\begin{tabular}{|c|c|c|c|c|c|}
\hline \multirow[b]{2}{*}{ Quality rank } & \multirow[b]{2}{*}{ Entry $^{z}$} & \multirow[b]{2}{*}{ Species } & \multicolumn{3}{|c|}{ Quality (1-9 scale) $)^{\mathrm{y}}$} \\
\hline & & & Overall & Yr 1 & Yr 2 \\
\hline 1 & PSG TH3 & Hard & 5.7 & 5.5 & 5.9 \\
\hline 2 & H575 Composite & Hard & 5.5 & 5.1 & 6.1 \\
\hline 3 & MEHI Composite (Gl) & Hard & 5.5 & 5.4 & 5.6 \\
\hline 4 & PPG-FL 108 & Hard & 5.5 & 5.3 & 5.7 \\
\hline 5 & MEH2 Composite (G1) & Hard & 5.4 & 5.3 & 5.6 \\
\hline 6 & 7Hl Composite & Hard & 5.4 & 5.3 & 5.6 \\
\hline 7 & BM2 Composite & Hard & 5.4 & 5.2 & 5.7 \\
\hline 8 & 7H4 Composite & Hard & 5.3 & 4.9 & 5.8 \\
\hline 9 & TEl Composite & Hard & 5.3 & 4.9 & 5.8 \\
\hline 10 & 7H3 Composite & Hard & 5.3 & 4.9 & 5.8 \\
\hline 11 & 4BND & Hard & 5.3 & 4.9 & 5.7 \\
\hline 12 & H573 Composite & Hard & 5.2 & 4.7 & 5.8 \\
\hline 13 & PPG-FL 107 & Hard & 5.2 & 4.9 & 5.5 \\
\hline 14 & Reliant IV & Hard & 5.2 & 4.8 & 5.7 \\
\hline 15 & MEWl Composite (Gl) & Chewings & 5.2 & 5.8 & 4.4 \\
\hline 16 & PPG-FL 106 & Hard & 5.2 & 4.7 & 5.8 \\
\hline 17 & TE2 Composite & Hard & 5.1 & 4.7 & 5.7 \\
\hline 18 & Marco Polo & Sheep & 5.1 & 5.1 & 5.2 \\
\hline 19 & Beacon & Hard & 5.1 & 4.6 & 5.7 \\
\hline 20 & Spartan II & Hard & 5.1 & 4.8 & 5.5 \\
\hline 21 & Blueray & Blue hard & 5.1 & 4.7 & 5.5 \\
\hline 22 & 7H2 Composite & Hard & 5.0 & 4.6 & 5.6 \\
\hline 23 & MEW2 Composite (Gl) & Chewings & 5.0 & 5.5 & 4.4 \\
\hline 24 & Predator & Hard & 5.0 & 4.4 & 5.7 \\
\hline 25 & H571 Composite & Hard & 4.9 & 4.3 & 5.6 \\
\hline 26 & MEW3 Composite (G1) & Chewings & 4.9 & 5.2 & 4.4 \\
\hline 27 & Radar & Chewings & 4.8 & 5.2 & 4.4 \\
\hline 28 & 7H6 Composite & Hard & 4.8 & 4.6 & 5.1 \\
\hline 29 & Soilguard & Hard & 4.6 & 4.2 & 5.0 \\
\hline 30 & Oxford & Hard & 4.6 & 4.0 & 5.3 \\
\hline 31 & Bighorn GT & Hard & 4.6 & 4.3 & 5.0 \\
\hline 32 & PPG-FRC 114 & Chewings & 4.5 & 4.8 & 4.3 \\
\hline 33 & PPG-FRC 115 & Chewings & 4.5 & 4.8 & 4.2 \\
\hline 34 & 7W3 Composite & Chewings & 4.5 & 4.7 & 4.2 \\
\hline 35 & 7Wl Composite & Chewings & 4.5 & 4.7 & 4.1 \\
\hline 36 & Fairmont & Chewings & 4.4 & 4.7 & 4.2 \\
\hline 37 & PPG-FRC 113 & Chewings & 4.4 & 4.5 & 4.3 \\
\hline 38 & PPG-FRC 107 & Chewings & 4.4 & 4.8 & 4.0 \\
\hline 39 & 7W4 Composite & Chewings & 4.3 & 4.8 & 3.8 \\
\hline 40 & Shadow II & Chewings & 4.3 & 4.5 & 4.2 \\
\hline 41 & SR5130 & Chewings & 4.2 & 4.4 & 4.1 \\
\hline 42 & Sealink & Slender creeping & 4.2 & 4.7 & 3.7 \\
\hline 43 & 2-10FRR-8 & Strong creeping & 4.2 & 4.2 & 4.2 \\
\hline 44 & Seabreeze GT & Slender creeping & 4.1 & 4.6 & 3.5 \\
\hline 45 & 08-5FCE+ & Chewings & 4.0 & 4.2 & 3.8 \\
\hline 46 & Compass & Chewings & 4.0 & 4.4 & 3.7 \\
\hline 47 & Daisy & Sheep & 4.0 & 3.8 & 4.3 \\
\hline 48 & Ambrose & Chewings & 4.0 & 4.2 & 3.8 \\
\hline 49 & 7W2 Composite & Chewings & 4.0 & 4.3 & 3.6 \\
\hline 50 & $4 \mathrm{SHR}-\mathrm{CH}$ & Chewings & 4.0 & 4.3 & 3.7 \\
\hline 51 & MESI Composite (Gl) & Strong creeping & 4.0 & 3.8 & 4.2 \\
\hline 52 & 2-10FRRBulk & Strong creeping & 4.0 & 4.0 & 3.9 \\
\hline 53 & 2-10FRR-6 & Strong creeping & 3.9 & 3.8 & 4.2 \\
\hline
\end{tabular}

(Continued on next page) 
Table 2. (Continued) Overall, year 1 and year 2 turfgrass quality ratings of first-generation mesotrione-tolerant selections, experimental selections, and commercially available cultivars of fine fescue in two field trials planted in 2013 and 2014 at the Rutgers Plant Biology Research and Extension Station in Adelphia, NJ. Mesotrione was applied at sowing (3 oz/acre + $0.25 \% \mathrm{v} / \mathrm{v}$ nonionic surfactant $)$ and $28 \mathrm{~d}$ after seedling emergence $(2 \mathrm{oz} / \mathrm{acre}+0.25 \% \mathrm{v} / \mathrm{v}$ nonionic surfactant $)$. Data were subjected to analysis of variance with the General Linear Model procedure in SAS (version 9.3; SAS Institute, Cary, NC). Means were separated with Fisher's protected least significant difference (LSD) test at $\alpha=0.05 .\left(1 \mathrm{oz} / \mathrm{acre}^{2}=70.0532 \mathrm{~g} \cdot \mathrm{ha}^{-1}\right)$.

\begin{tabular}{|c|c|c|c|c|c|}
\hline \multirow[b]{2}{*}{ Quality rank } & \multirow[b]{2}{*}{ Entry $^{\mathrm{z}}$} & \multirow[b]{2}{*}{ Species } & \multicolumn{3}{|c|}{ Quality $(1-9 \text { scale })^{y}$} \\
\hline & & & Overall & Yr 1 & Yr 2 \\
\hline 54 & $4 \mathrm{CHY}$ & Chewings & 3.9 & 4.2 & 3.7 \\
\hline 55 & MES2 Composite (G1) & Strong creeping & 3.8 & 3.8 & 3.9 \\
\hline 56 & Azure & Hard & 3.8 & 3.5 & 4.2 \\
\hline 57 & 2-10FRR-12 & Strong creeping & 3.8 & 3.7 & 3.9 \\
\hline 58 & 2-10FRR-4 & Strong creeping & 3.8 & 3.7 & 4.0 \\
\hline 59 & S571 Composite & Strong creeping & 3.8 & 3.7 & 3.9 \\
\hline 60 & Navigator II & Strong creeping & 3.8 & 3.8 & 3.7 \\
\hline 61 & SR5250 & Strong creeping & 3.7 & 3.7 & 3.8 \\
\hline 62 & 7C3 Composite & Strong creeping & 3.7 & 3.5 & 3.9 \\
\hline 63 & 2-10FRR-13 & Strong creeping & 3.7 & 3.7 & 3.8 \\
\hline 64 & PPG-FRR 111 & Strong creeping & 3.7 & 3.8 & 3.6 \\
\hline 65 & Cardinal & Strong creeping & 3.7 & 3.7 & 3.7 \\
\hline 66 & Jasper II & Strong creeping & 3.7 & 3.7 & 3.7 \\
\hline 67 & Seafire & Slender creeping & 3.7 & 3.9 & 3.4 \\
\hline 68 & OR 126 & Strong creeping & 3.7 & 3.7 & 3.6 \\
\hline 69 & CRF-11-4A & Strong creeping & 3.6 & 3.7 & 3.4 \\
\hline 70 & PPG-FRR 103 & Strong creeping & 3.6 & 3.6 & 3.5 \\
\hline 71 & 4-12FF-Bulk & Strong creeping & 3.5 & 3.9 & 3.2 \\
\hline 72 & FRR 62 & Strong creeping & 3.5 & 3.4 & 3.7 \\
\hline 73 & PSG $50 \mathrm{C} 3$ & Chewings & 3.5 & 3.4 & 3.6 \\
\hline 74 & Windward & Chewings & 3.4 & 3.6 & 3.3 \\
\hline 75 & Kent & Strong creeping & 3.4 & 3.6 & 3.2 \\
\hline 76 & FT5 Composite & Strong creeping & 3.4 & 3.3 & 3.6 \\
\hline 77 & $4-12 \mathrm{FF}-3^{1}$ & Strong creeping & 3.4 & 3.7 & 3.1 \\
\hline 78 & $5-12 \mathrm{FF}-8$ & Strong creeping & 3.4 & 3.6 & 3.2 \\
\hline 79 & Shademaster III & Strong creeping & 3.4 & 3.4 & 3.4 \\
\hline 80 & FT2 Composite & Strong creeping & 3.4 & 3.4 & 3.5 \\
\hline 81 & BRSG & Strong creeping & 3.4 & 3.6 & 3.2 \\
\hline 82 & BRSO & Strong creeping & 3.4 & 3.5 & 3.3 \\
\hline 83 & Gibraltar & Strong creeping & 3.4 & 3.4 & 3.3 \\
\hline 84 & $\mathrm{FF} 2$ & Strong creeping & 3.3 & 3.5 & 3.2 \\
\hline 85 & PSG 5RM & Strong creeping & 3.3 & 3.3 & 3.4 \\
\hline 86 & $4-12 \mathrm{FF}-1$ & Strong creeping & 3.3 & 3.6 & 3.0 \\
\hline 87 & FT6 Composite & Strong creeping & 3.3 & 3.2 & 3.5 \\
\hline 88 & FT4 Composite & Strong creeping & 3.3 & 3.2 & 3.5 \\
\hline 89 & Miser & Strong creeping & 3.3 & 3.2 & 3.5 \\
\hline 90 & FT3 Composite & Strong creeping & 3.3 & 3.0 & 3.6 \\
\hline 91 & $4-12 \mathrm{FF}-2$ & Strong creeping & 3.3 & 3.6 & 2.9 \\
\hline 92 & $4-12 F F-5$ & Strong creeping & 3.3 & 3.6 & 2.9 \\
\hline 93 & Gibraltar Gold & Strong creeping & 3.2 & 3.2 & 3.3 \\
\hline 94 & 4CRD- 8 & Strong creeping & 3.2 & 3.3 & 3.1 \\
\hline 95 & 4CRD-P & Strong creeping & 3.2 & 3.2 & 3.3 \\
\hline 96 & $5-12$ FF- 6 & Strong creeping & 3.2 & 3.1 & 3.3 \\
\hline 97 & FT1 Composite & Strong creeping & 3.2 & 3.0 & 3.4 \\
\hline 98 & $5-12 \mathrm{FF}-5$ & Strong creeping & 3.2 & 3.4 & 2.9 \\
\hline 99 & Oracle & Strong creeping & 3.0 & 3.0 & 3.0 \\
\hline 100 & 4GRP & Strong creeping & 3.0 & 3.0 & 3.0 \\
\hline 101 & 5-12FF-Bulk & Strong creeping & 3.0 & 2.9 & 3.1 \\
\hline 102 & $5-12 \mathrm{FF}-4$ & Strong creeping & 2.4 & 2.2 & 2.8 \\
\hline \multirow[t]{2}{*}{103} & 7 Seas & Chewings & 1.4 & 1.3 & 1.5 \\
\hline & & $\operatorname{LSD}(\alpha=0.05)$ & 0.5 & 0.7 & 0.5 \\
\hline
\end{tabular}

${ }^{2} \mathrm{Gl}$ in parentheses indicates first-generation mesotrione-tolerant selection.

${ }^{\mathrm{y}} \mathrm{l}=$ lowest quality, $5=$ minimum acceptable rating, and $9=$ best turfgrass quality 
Table 3. The effect of mesotrione on the establishment and injury ratings of first- and second-generation mesotrione-tolerant selections, experimental selections, and commercially available cultivars of fine fescue in a field trial planted in 2015 at the Rutgers Plant Biology Research and Extension Station in Adelphia, NJ. Mesotrione was applied at 3 oz/acre at sowing and $28 \mathrm{~d}$ after seedling emergence $+\mathbf{0 . 2 5 \%} \mathrm{v} / \mathrm{v}$ nonionic surfactant. Data were subjected to analysis of variance with the General Linear Model procedure in SAS (version 9.3; SAS Institute, Cary, NC). Means were separated with Fisher's protected least significant difference (LSD) test at $\alpha=0.05$. (1 oz/acre $\left.=70.0532 \mathrm{~g} \cdot \mathrm{ha}^{-1}\right)$.

\begin{tabular}{|c|c|c|c|c|}
\hline Injury rank & Entry $^{z}$ & Species & Establishment (1-9 scale $)^{\mathrm{y}}$ & Injury (1-9 scale $)^{x}$ \\
\hline 1 & TEH2 Composite (G2) & Hard & 8.0 & 8.2 \\
\hline 2 & TEH4 Composite (G2) & Hard & 7.3 & 8.2 \\
\hline 3 & TEH3 Composite (G2) & Hard & 7.7 & 8.0 \\
\hline 4 & TEH1 Composite (G2) & Hard & 7.7 & 7.8 \\
\hline 5 & TW2 Composite (G2) & Chewings & 7.0 & 7.7 \\
\hline 6 & Minimus & Hard & 5.7 & 7.2 \\
\hline 7 & TW1 Composite (G2) & Chewings & 5.3 & 7.0 \\
\hline 8 & MEHl Composite (Gl) & Hard & 6.3 & 6.2 \\
\hline 9 & Chariot & Hard & 5.3 & 6.2 \\
\hline 10 & Firefly & Hard & 4.0 & 6.2 \\
\hline 11 & MEWl Composite (Gl) & Chewings & 7.0 & 6.2 \\
\hline 12 & Sword & Hard & 5.0 & 6.0 \\
\hline 13 & TRl Composite (G2) & Strong creeping & 6.0 & 6.0 \\
\hline 14 & MEH2 Composite (Gl) & Hard & 6.0 & 5.5 \\
\hline 15 & FH2 Composite & Hard & 4.7 & 5.3 \\
\hline 16 & FH3 Composite & Hard & 5.3 & 5.3 \\
\hline 17 & Beacon & Hard & 4.0 & 5.3 \\
\hline 18 & Radar & Chewings & 5.0 & 5.3 \\
\hline 19 & MEW2 Composite (Gl) & Chewings & 7.3 & 5.0 \\
\hline 20 & FH4 Composite & Hard & 4.7 & 4.8 \\
\hline 21 & PPG-FRC 113 & Chewings & 4.7 & 4.8 \\
\hline 22 & Compass & Chewings & 4.3 & 4.7 \\
\hline 23 & Blueray & Blue hard & 4.3 & 4.3 \\
\hline 24 & TR2 Composite (G2) & Strong creeping & 5.0 & 4.3 \\
\hline 25 & FW2 Composite & Chewings & 4.7 & 4.2 \\
\hline 26 & FR3 Composite & Strong creeping & 4.0 & 4.2 \\
\hline 27 & FHl Composite & Hard & 3.7 & 4.0 \\
\hline 28 & Blue Hornet & Sheep & 3.0 & 3.2 \\
\hline 29 & Ambrose & Chewings & 2.3 & 3.2 \\
\hline 30 & Marvel & Strong creeping & 3.0 & 3.2 \\
\hline 31 & Lighthouse & Slender creeping & 3.0 & 3.0 \\
\hline 32 & PPG-FRT 101 & Slender creeping & 3.7 & 3.0 \\
\hline 33 & PPG-FRR 111 & Strong creeping & 3.0 & 3.0 \\
\hline 34 & Garnet & Strong creeping & 2.3 & 2.8 \\
\hline 35 & FRl Composite & Strong creeping & 2.3 & 2.5 \\
\hline 36 & FR2 Composite & Strong creeping & 2.0 & 2.5 \\
\hline 37 & Navigator II & Strong creeping & 2.3 & 2.5 \\
\hline 38 & Pathfinder & Strong creeping & 2.7 & 2.5 \\
\hline 39 & FR4 Composite & Strong creeping & 2.3 & 2.3 \\
\hline 40 & Cardinal & Strong creeping & 2.7 & 2.3 \\
\hline 41 & MES2 Composite (Gl) & Strong creeping & 3.3 & 2.0 \\
\hline 42 & SR5250 & Strong creeping & 1.7 & 1.8 \\
\hline 43 & Predator & Hard & 2.3 & 1.5 \\
\hline 44 & PPG-FL 106 & Hard & 2.0 & 1.3 \\
\hline 45 & Audubon & Strong creeping & 1.3 & 1.2 \\
\hline 46 & Reliant IV & Hard & 1.7 & 1.0 \\
\hline 47 & Rescue 911 & Hard & 1.3 & 1.0 \\
\hline \multirow[t]{2}{*}{48} & Azure & Hard & 1.3 & 1.0 \\
\hline & & $\operatorname{LSD}(\alpha=0.05)$ & 1.0 & 2.1 \\
\hline
\end{tabular}

${ }^{\mathrm{z}} \mathrm{Gl}$ or $\mathrm{G} 2$ in parentheses indicates first- or second-generation mesotrione-tolerant selection, respectively.

${ }^{y}$ Mesotrione effects on establishment compared with untreated, where $1=$ complete inhibition of establishment, $5=$ minimum acceptable rating, and $9=$ no reduction in establishment.

${ }^{\mathrm{x}}$ Injury on a 1 - to 9 -point scale, with $\mathrm{l}=$ completely bleached tissue, $5=$ minimum acceptable rating, and $9=$ no injury 
Table 4. Turf quality ratings of mesotrione-treated strip $(3 \mathrm{oz} / \mathrm{acre}+0.25 \% \mathrm{v} / \mathrm{v}$ nonionic surfactant applications at sowing and $28 \mathrm{~d}$ after seedling emergence) and untreated strip of first- and second-generation mesotrione-tolerant selections, commercially available cultivars, and experimental selections of fine fescues in a field trial planted in Sept. 2015 at the Rutgers Plant Biology Research and Extension Station in Adelphia, NJ. Data were subjected to analysis of variance with the General Linear Model procedure in SAS (version 9.3; SAS Institute, Cary, NC). Means were separated with Fisher's protected least significant difference (LSD) test at $\alpha=0.05 .\left(1 \mathrm{oz} / \mathrm{acre}=70.0532 \mathrm{~g} \cdot \mathrm{ha}^{-1}\right)$.

\begin{tabular}{|c|c|c|c|c|c|}
\hline \multirow[b]{2}{*}{ Injury rank } & \multirow[b]{2}{*}{ Entry $^{z}$} & \multirow[b]{2}{*}{ Species } & \multicolumn{3}{|c|}{ Quality (1-9 scale) } \\
\hline & & & 2016 Mesotrione & 2016 Untreated & $2017^{x}$ \\
\hline 1 & TEH2 Composite (G2) & Hard & 5.7 & 5.6 & 6.3 \\
\hline 2 & TEH4 Composite (G2) & Hard & 5.8 & 5.7 & 6.4 \\
\hline 3 & TEH3 Composite (G2) & Hard & 5.9 & 5.8 & 6.5 \\
\hline 4 & TEHl Composite (G2) & Hard & 6.0 & 5.9 & 6.1 \\
\hline 5 & TW2 Composite (G2) & Chewings & 5.2 & 5.2 & 4.8 \\
\hline 6 & Minimus & Hard & 5.0 & 5.0 & 5.0 \\
\hline 7 & TW1 Composite (G2) & Chewings & 5.2 & 5.1 & 4.6 \\
\hline 8 & MEHI Composite (Gl) & Hard & 5.5 & 5.4 & 5.4 \\
\hline 9 & Chariot & Hard & 5.1 & 5.0 & 5.2 \\
\hline 10 & Firefly & Hard & 4.7 & 4.7 & 5.4 \\
\hline 11 & MEWl Composite (Gl) & Chewings & 5.5 & 5.4 & 4.8 \\
\hline 12 & Sword & Hard & 4.9 & 4.9 & 5.0 \\
\hline 13 & TRl Composite (G2) & strong creeping & 5.0 & 4.9 & 3.7 \\
\hline 14 & MEH2 Composite (G1) & Hard & 5.7 & 5.9 & 6.3 \\
\hline 15 & FH2 Composite & Hard & 4.8 & 5.0 & 5.8 \\
\hline 16 & FH3 Composite & Hard & 4.5 & 4.8 & 5.3 \\
\hline 17 & Beacon & Hard & 4.9 & 5.0 & 5.3 \\
\hline 18 & Radar & Chewings & 4.7 & 4.6 & 4.4 \\
\hline 19 & MEW2 Composite (Gl) & Chewings & 5.3 & 5.1 & 4.5 \\
\hline 20 & FH4 Composite & Hard & 5.3 & 5.2 & 6.1 \\
\hline 21 & PPG-FRC 113 & Chewings & 5.0 & 4.9 & 4.8 \\
\hline 22 & Compass & Chewings & 4.1 & 4.1 & 3.6 \\
\hline 23 & Blueray & Blue hard & 4.0 & 4.1 & 3.9 \\
\hline 24 & TR2 Composite (G2) & Strong creeping & 5.5 & 5.4 & 4.1 \\
\hline 25 & FW2 Composite & Chewings & 5.5 & 5.4 & 5.0 \\
\hline 26 & FR3 Composite & Strong creeping & 4.7 & 4.8 & 4.4 \\
\hline 27 & FHl Composite & Hard & 5.3 & 5.4 & 6.6 \\
\hline 28 & Blue Hornet & Sheep & 3.2 & 3.3 & 3.6 \\
\hline 29 & Ambrose & Chewings & 4.1 & 4.2 & 3.7 \\
\hline 30 & Marvel & Strong creeping & 3.6 & 3.9 & 3.1 \\
\hline 31 & Lighthouse & slender creeping & 2.7 & 2.6 & 2.6 \\
\hline 32 & PPG-FRT 101 & slender creeping & 4.8 & 5.0 & 5.0 \\
\hline 33 & PPG-FRR 111 & Strong creeping & 4.3 & 4.2 & 3.8 \\
\hline 34 & Garnet & Strong creeping & 2.6 & 3.4 & 3.0 \\
\hline 35 & FRl Composite & Strong creeping & 4.1 & 4.4 & 4.8 \\
\hline 36 & FR2 Composite & Strong creeping & 4.4 & 4.9 & 4.7 \\
\hline 37 & Navigator II & Strong creeping & 3.4 & 4.0 & 3.3 \\
\hline 38 & Pathfinder & Strong creeping & 3.1 & 3.4 & 3.1 \\
\hline 39 & FR4 Composite & Strong creeping & 4.6 & 4.8 & 4.8 \\
\hline 40 & Cardinal & Strong creeping & 3.4 & 3.7 & 3.2 \\
\hline 41 & MES2 Composite (Gl) & Strong creeping & 4.8 & 5.2 & 3.7 \\
\hline 42 & SR5250 & Strong creeping & 2.5 & 3.2 & 2.9 \\
\hline 43 & Predator & Hard & 3.8 & 4.1 & 5.2 \\
\hline 44 & PPG-FL 106 & Hard & 2.6 & 3.5 & 4.6 \\
\hline 45 & Audubon & Strong creeping & 2.0 & 3.3 & 3.3 \\
\hline 46 & Reliant IV & Hard & 3.4 & 3.9 & 5.5 \\
\hline 47 & Rescue 911 & Hard & 2.0 & 2.3 & 3.1 \\
\hline \multirow[t]{2}{*}{48} & Azure & Hard & 2.0 & 2.7 & 3.5 \\
\hline & & $\operatorname{LSD}(\alpha=0.05)$ & 1.1 & 0.9 & 0.9 \\
\hline
\end{tabular}

${ }^{\mathrm{z}} \mathrm{G} 1$ or $\mathrm{G} 2$ in parentheses indicates first- or second-generation mesotrione-tolerant selection, respectively.

${ }^{\mathrm{y}}$ Quality rated on a 1 - to 9 -point scale, with $\mathrm{l}=$ lowest quality, $5=$ minimum acceptable rating, and $9=$ best turfgrass quality

${ }^{x}$ Plots had no effect from herbicide application so the entire plot was rated for turf quality. 
establishment ratings were relatively poor, with ratings of 2.8 and 3.0, respectively. The three Gl mesotrione-tolerant selections of chewings fescues (MEW1 Composite, MEW2 Composite, and MEW3 Composite) had good establishment overall, with ratings of $5.2,5.3$, and 4.9 , respectively. The Gl mesotrione-tolerant selections of strong creeping red fescues (MES1 Composite and MES2 Composite) had establishment ratings of 3.2 and 3.6, respectively. The strong creeping red fescues were slower to establish compared with other fine fescue field trials conducted at the Adelphia farm for those years that were not treated with mesotrione, and it was suspected that the mesotrione applications could have been a factor.

Turf quality was analyzed for each year (year 1 and year 2) and overall for both years (Table 2). Entries that were poor to establish in general had poor turf quality ratings for year 1 , but some of those had much better year 2 turf quality, which indicates there could have been lasting effects from the mesotrione treatments that never allowed the grasses to establish fully. Having treated and untreated sections of each plot would allow one to observe more comprehensively the effects of the herbicide compared with the genetics of each cultivar in the next-generation field trial. Overall, the Gl mesotrionetolerant selections had acceptable turf quality or a turf quality rating that was better than the species average.

G2 FIELD TRIAL. Overall, the performance of the G2 mesotrionetolerant selections were better than the Gl within each species. The four G2 mesotrione-tolerant selections of hard fescue (TEH4 Composite, TEH2 Composite,TEH3 Composite, and TEHl Composite) were the least injured in the trial, with ratings of $8.2,8.2,8.0$, and 7.8 , respectively (Table 3). The mesotrione-tolerant selections of chewings fescue (TW2 Composite and TWl Composite) had very little injury, with ratings of 7.7 and 7.0, respectively, compared with the Gl MEWl Composite and MEW2 Composite, which rated 6.2 and 5.0, respectively. All the G2 hard and chewings selections were in the top statistical grouping for injury. The G2 mesotrione-tolerant selections of strong creeping red fescues (TRI Composite and TR2 Composite) were not in the top statistical group, but only had moderate injury $(6.0$ and 4.3 ) and were better than the Gl strong creeping red fescue MES2 Composite, which rated a 4.0.

Establishment ratings ranged from 8.0 to 1.3 ( LSD $=1.0$ ) (Table $3)$. The G2 mesotrione-tolerant selections of hard fescue (TEH2 Composite, TEH3 Composite, TEHI Composite, and TEH4 Composite) were least affected by mesotrione at establishment and were the top rated, with ratings of $8.0,7.7,7.7$, and 7.3 , respectively. This was significantly greater than the Gl hard fescues, which had ratings of 6.3 and 6.0 . The G2 mesotrione-tolerant chewings selections were affected minimally by mesotrione at establishment and had better establishment than the two Gl chewings fescue composites. The G2 strong creeping red fescues had much better establishment compared with Gl: TRl Composite rating of 6.0 and TR2 Composite rating of 5.0 compared with the G1 MES2 Composite rating of 3.3 .

Turf quality was affected by the mesotrione applications in some of the more sensitive entries, so ratings were taken for both the control strips and the mesotrione-treated strips separately for year 1 . Turf quality for the year 1 nonmesotrione-treated control ranged from 2.3 to 5.9, with an LSD of 0.9 (Table 4). For the mesotrionetreated strip, turf quality ranged from 2.0 to 6.0 , with an LSD of 1.1. The mesotrione-tolerant selections from the G2 and G1 entries all maintained acceptable turf quality ratings, with all the $\mathrm{G} 2$ entries being in the top statistical group, excluding TRl Composite, which was just 0.1 less than the cutoff for top statistical grouping.

By year 2, there were no visual effects from the mesotrione treatments, so entire plots were rated for turf quality (Table 4 ). The turf quality ratings for year 2 of the trial ranged from 2.6 to 6.6 , with an LSD of 0.9. The top statistical grouping for year 2 turf quality ratings included all four G2 mesotrione-tolerant selection hard fescues and the MEH2 Composite from the Gl entries. Progress was made in increasing the tolerance to mesotrione from G1 to G2 while maintaining and, in many cases, improving the turfgrass quality.

G3 FIELD TRIAL. The range of injury and establishment ratings for the 4 -oz/acre rate was 1.4 to 9.0 , with an LSD of 0.9 (Table 5 ). The top statistical grouping for the 4-oz/acre rate included all the G3 mesotrionetolerant selections for all three species. The HTB5 Composite (hard fescue) was the best-performing entry in the trial for injury and establishment. For the $8-\mathrm{oz} /$ acre rate, the rating ranged from 1.0 to 8.3 , with an LSD of 1.3 . The top statistical grouping of the $8-\mathrm{oz} /$ acre rate included all the G3 hard fescues, all the G3 chewings fescues, and the G3 strong creeping red fescue STBI Composite, which ranked fifth overall in each rating. This was interesting and exciting to see because, from G1 to G2, the strong creeping red fescues had the least amount of improvement.

Plants from the Gl progeny were selected to determine whether absorption and translocation were associated with bleaching injury. Studies were conducted using foliar and root uptake of carbon 14 radiolabeled isotope (14C) mesotrione along with a rate titration of three lines each of chewings fescue, hard fescue, and strong creeping red fescue (Tate et al., 2019). Translocation of foliarabsorbed 14C mesotrione was not associated with differential tolerance levels of the three species. There were differences among lines within species for root absorption, but it was comparable among the three species. This suggests factors other than absorption and translocation are what is being selected for in recurrent breeding, and further studies are needed to determine the mechanism for increased tolerance.

\section{Conclusions}

These results demonstrate that using a recurrent selection method is an effective way to increase the tolerance of fine fescues to mesotrione herbicide. After three generations, selections of hard, chewings, and strong creeping red fescues had equivalent or better tolerance to mesotrione than tall fescues and kentucky bluegrasses, which are on the label for safe use at seeding. These G3 selections will continue to be tested for their turf quality performance and to ensure there are not any long-term adverse effects of mesotrione on these species. If they continue to do well in testing, the G3 fine fescues could be treated safely with mesotrione at seeding in the future to establish 
Table 5. Injury ratings at two rates of mesotrione-treated first-, second-, and third-generation mesotrione-tolerant selections, experimental selections, and commercially available cultivars of fine fescues, tall fescues, and kentucky bluegrasses in a field trial planted in Sept. 2018 at the Rutgers Plant Biology Research and Extension Station in Adelphia, NJ. Mesotrione was applied at 4 - and 8 -oz/acre rates $+0.25 \% \mathrm{v} / \mathrm{v}$ nonionic surfactant at sowing and $28 \mathrm{~d}$ after seedling emergence. Data were subjected to analysis of variance with the General Linear Model procedure in SAS (version 9.3; SAS Institute, Cary, NC). Means were separated with Fisher's protected least significant difference (LSD) test at $\alpha=0.05 .\left(1 \mathrm{oz} / \mathrm{acre}^{2}=70.0532 \mathrm{~g} \cdot \mathrm{ha}^{-1}\right)$.

\begin{tabular}{|c|c|c|c|c|}
\hline \multirow[b]{2}{*}{ Injury rank } & \multirow[b]{2}{*}{ Cultivar $^{\mathrm{z}}$} & \multirow[b]{2}{*}{ Species } & \multicolumn{2}{|c|}{ Injury (1-9 scale) $)^{y}$} \\
\hline & & & $0.25 \mathrm{lb} / \mathrm{acre}$ & $0.5 \mathrm{lb} / \mathrm{acre}$ \\
\hline 1 & HTB5 Composite (G3) & Hard & 9.0 & 8.3 \\
\hline 2 & HTB4 Composite (G3) & Hard & 8.9 & 8.3 \\
\hline 3 & TEH3 Composite (G2) & Hard & 8.9 & 8.3 \\
\hline 4 & TEH2 Composite (G2) & Hard & 9.0 & 8.1 \\
\hline 5 & STB1 Composite (G3) & Strong creeping & 8.8 & 8.1 \\
\hline 6 & WTBT1 Composite (G3) & Chewings & 8.9 & 7.9 \\
\hline 7 & WTB2 Composite (G3) & Chewings & 8.9 & 7.9 \\
\hline 8 & Padre II & Tall fescue & 8.5 & 7.9 \\
\hline 9 & WTBT3 Composite (G3) & Chewings & 8.9 & 7.7 \\
\hline 10 & WTU5 Composite (G3) & Chewings & 8.9 & 7.7 \\
\hline 11 & Talladega & Tall fescue & 8.3 & 7.6 \\
\hline 12 & Bordeaux & Kentucky bluegrass & 8.3 & 7.5 \\
\hline 13 & HTN3 Composite (G3) & Hard & 8.9 & 7.5 \\
\hline 14 & STU2 Composite (G3) & Strong creeping & 8.5 & 7.5 \\
\hline 15 & HTB2 Composite (G3) & Hard & 8.7 & 7.4 \\
\hline 16 & WTB4 Composite (G3) & Chewings & 8.6 & 7.3 \\
\hline 17 & Champagne & Kentucky bluegrass & 8.1 & 7.1 \\
\hline 18 & HTB1 Composite (G3) & Hard & 8.7 & 7.1 \\
\hline 19 & Gladiator & Hard & 8.7 & 7.0 \\
\hline 20 & Hot Rod & Tall fescue & 8.5 & 7.0 \\
\hline 21 & TW2 Composite (G2) & Chewings & 8.3 & 6.9 \\
\hline 22 & Sword & Hard & 8.3 & 6.7 \\
\hline 23 & STB3 Composite (G3) & Strong creeping & 8.3 & 6.7 \\
\hline 24 & TRI Composite (G2) & Strong creeping & 7.9 & 6.7 \\
\hline 25 & STN4 Composite (G3) & Strong creeping & 8.5 & 6.6 \\
\hline 26 & Blue Note & Kentucky bluegrass & 7.5 & 6.5 \\
\hline 27 & Windward & Chewings & 8.0 & 6.5 \\
\hline 28 & Ambrose & Chewings & 8.1 & 6.3 \\
\hline 29 & Marvel & Strong creeping & 7.3 & 5.5 \\
\hline 30 & MEWl Composite (Gl) & Chewings & 6.9 & 4.4 \\
\hline 31 & Fairmont & Chewings & 7.6 & 4.3 \\
\hline 32 & Navigator II & Strong creeping & 6.8 & 4.2 \\
\hline 33 & MEH2 Composite (Gl) & Hard & 5.1 & 3.3 \\
\hline 34 & MEHl Composite (Gl) & Hard & 5.5 & 2.5 \\
\hline 35 & SR5250 & Strong creeping & 2.6 & 1.3 \\
\hline \multirow{2}{*}{36} & Garnet & Strong creeping & 1.4 & 1.0 \\
\hline & & $\operatorname{LSD}(\alpha=0.05)$ & 0.9 & 1.3 \\
\hline
\end{tabular}

${ }^{\mathrm{z}} \mathrm{G} 1, \mathrm{G} 2$, or G3 in parentheses indicates first-, second-, or third-generation mesotrione-tolerant selection, respectively.

${ }^{\mathrm{y}} \mathrm{l}=$ major reduction in establishment (i.e., bare soil) and completely bleached tissue, $5=$ minimum acceptable rating, and $9=$ no injury or reduction in establishment.

low-maintenance turf that is free from weed competition during establishment. This research helps solve a critical need in the industry and should increase the use of fine fescues in the industry by providing a tool to help establish these grasses while controlling problematic weeds such as annual bluegrass and other broadleaf weeds. Using mesotrione does not complicate or reduce the control options for these fine fescues because there are still many effective herbicides and classes of herbicides that still provide effective control. Future research is planned to determine whether mesotrione can be used safely for weed control for seed production. If that research shows positive results, and a special use label is granted, then it would give seed farmers of these grasses another tool for weed control as well.

\section{Literature cited}

Askew, S.D. and J.B. Beam. 2002. Weed management in cool-season turf with mesotrione. Proc. Northeast Weed Sci. Soc. 56:129.
Beard, J.B. 1973. Turfgrass: Science and culture. Prentice Hall, Englewood Cliffs, NJ.

Grande, J. 2004. Seeding your lawn. Rutgers Coop. Res. Ext. fact sheet FS584.

Hanson, A.A., F.V. Juska, and G.W Burton. 1969. Species and varieties, p 370-409. In: A.A. Hanson and F.V. Juska (eds.). Turfgrass science. American Society of Agronomy, Madison, WI.

Hart, S.E., J.F. Derr, D.W. Lycan, C. Rose-Fricker, and W.A. Meyer. 2005. Increased glyphosate tolerance in 'Aurora 
Gold' hard fescue (Festuca longifolia). Weed Technol. 19:640-646, doi: 10.1614/WT-04-200R.1.

Hart, S.E., P. McCullough, and C.J. Mansue. 2007. Use of mesotrione herbicide for weed control at cool-season turfgrass establishment. Ctr. Turfgrass Sci. Proc. 35-36.

Johnston, D.T. and J.S. Faulkner. 1986. Countess and Duchess: Aminotriazoletolerant cultivars of chewings fescue and browntop bent. J. Sports Turf Res. Inst. 62:217.

Kaminski, J.E. and P.H. Dernoeden. 2007. Seasonal Poa annua L. seedling emergence patterns in Maryland. Crop Sci. 47:775-779, doi: 10.2135/cropsci2006. 03.0191 .

McCullough, P.E., J. Yu, D.G. Shilling, and M.A. Czarnota. 2015. Physiological basis for glyphosate tolerance in hard fescue and perennial ryegrass cultivars. Crop Sci. 55:2352-2358, doi: 10.2135/ cropsci2015.01.0035.

Meyer, W.A. and C.R. Funk. 1989. Progress and benefits to humanity from breeding cool-season grasses for turf, $\mathrm{p}$. 31-48. In: D.A. Sleper, K.H. Asay, and
J.F. Pedersen (eds.). Contributions from breeding forage and turf grasses. Crop Science Society of America, Madison, WI

Musser, H.B. and A.T. Perkins. 1969 Guide to seedbed preparation, p. 462 473. In: A.A. Hanson and F.V. Juska (eds.). Turfgrass science. American Society of Agronomy, Madison, WI.

Pavlick, L.E. 1985. A new taxonomic survey of the Festuca rubra complex in northwestern North America, with emphasis on British Columbia. Phytologia 57:1-17.

Ruemmele, B.A., J.K. Wipff, L. Brilman, and K.W. Hignight. 2003. Fine-leaved Festuca species, p. 129-174. In: M.D. Cassler and R.R. Duncan (eds.). Turfgrass biology, genetics, and breeding. Wiley, Hoboken, NJ.

Syngenta Crop Protection. 2008. Tenacity $^{\circledR}$ product label. Syngenta Corp Protection, Greensboro, NC.
Tate, T.M., W.A. Meyer, P.E. McCullough, and J. Yu. 2019. Evaluation of mesotrione tolerance levels and [14C] mesotrione absorption and translocation in three fine fescue species. Weed Sci. 67:497-503, doi: 10.1017/wsc.2019.39.

Turgeon, A. 1996. Turfgrass management. Prentice Hall, Upper Saddle River, NJ.

Vogel, K. and J.F. Pedersen. 1993. Breeding systems for cross-pollinated perennial grasses. Plant Breed. Rev. 11:251274.

Williams, M.W., W.J. Johnston, J.P. Yenish, E.D. Miltner, and C.T. Golob. 2009. Glasshouse evaluation of pre-plant and at planting applications of mesotrione on perennial ryegrass and chewings fescue. Intl. Turfgrass Soc. Res. J. 11(part 2):1237-1245.

Willis, J.B., J.B. Beam, W.L. Barker, and S.D. Askew. 2006. Weed control options in spring-seeded tall fescue (Festuca arundinacea). Weed Technol. 4:10401046, doi: 10.1614/WT-05-138.1. 